\title{
Crystallization and mechanical properties of biodegradable poly(p-dioxanone)/octamethyl-polyhedral oligomeric silsesquioxanes nanocomposites via simple solution casting method
}

\author{
ZHECUN WANG ${ }^{1,2}$, CHENGDONG XIONG ${ }^{1}$ and QING LI ${ }^{1, *}$ \\ ${ }^{1}$ Chengdu Institute of Organic Chemistry, Chinese Academy of Sciences, Chengdu, Sichuan 610041, China \\ ${ }^{2}$ University of Chinese Academy of Sciences, Beijing 10049, China
}

MS received 13 May 2015; accepted 11 June 2015

\begin{abstract}
In this study, biodegradable poly(p-dioxanone) (PPDO)/octamethyl-polyhedral oligomeric silsesquioxanes (ome-POSS) nanocomposites were fabricated by the simple solution casting method with various ome-POSS loadings. Scanning electron microscopic observations indicate that ome-POSS is well dispersed in the PPDO matrix. Effect of ome-POSS on the isothermal melt crystallization and dynamic mechanical properties of PPDO in the nanocomposites were studied in detail. It shows that the overall crystallization rates are faster in the nanocomposites than in neat PPDO and increase with the increase in ome-POSS loadings; however, X-ray diffraction patterns, POM and the Avrami exponent suggest that the crystal structure and the crystallization mechanism do not change despite the presence of ome-POSS. The mechanical property of PPDO/ome-POSS nanocomposites was enhanced with respect to neat PPDO.
\end{abstract}

Keywords. Poly(p-dioxanone); octamethyl-polyhedral oligomeric silsesquioxanes; crystallization.

\section{Introduction}

Poly(p-dioxanone) (PPDO), a typical linear thermoplastic aliphatic poly(ester-ether) with outstanding biodegradability, flexibility and biocompatibility, is widely used as suture material. ${ }^{1}$ However, PPDO is easily degradable in the ambient environment and has the poor mechanical properties (especially the modulus), which leads to the great limitation of the availability of PPDO as other medical devices. ${ }^{2}$ Except chemical structure, the crystal structure and crystallinity can also influence the mechanical property and degradation behaviour of PPDO. For this reason, the crystal structure, isothermal crystallization and nonisothermal crystallization were widely studied in recent times. ${ }^{3-8}$ The crystallization of PPDO was enhanced with different nucleating agents. The isothermal crystallization of PPDO was studied in detail in the presence of different additives and found that the acceleration effects of nucleating agents on the overall crystallization of PPDO decrease in the following order: $\mathrm{BN} \geq$ talc $\geq \mathrm{HA}^{.}{ }^{5}$ In recent times, there are also some interesting developments on the crystallization of PPDO. Compared to the poly(lactide acid)/PDDO homopolymer blend, the growth rate and nucleation rate of PPDO were enhanced rather than depressed when blended with branched PPDO-PLA block copolymer. ${ }^{9}$ Of particular interest is

\footnotetext{
*Author for correspondence (zhecun1988@126.com)
}

the nanocomposite technology developed in recent times consisting of synthetic hectorite and montmorillonite. ${ }^{10,11}$ The nanocomposites were prepared successfully by in situ ring-opening polymerization, moreover the crystallization and mechanical properties of PPDO were improved in the nanocomposites.

Polyhedral oligomeric silsesquioxane (POSS) is a new three-dimensional nanofiller which can be introduced to polymer by copolymerization or blending to prepare the highperformance nanostructured organic-inorganic composites attracting more and more attention in recent times. ${ }^{12-14}$ Blending with other material is easy and inexpensive way to prepare the nanocomposites, and then lots of polymers blended with POSS have been reported via different methods. ${ }^{15-19}$ Biodegradable polymer nanocomposites comprising of POSS by physical blending have attracted much more attention in recent times. PLLA/POSS were prepared by the solution casting method, solution and coagulation method, and simple melt compounding method. It is found that the POSS is dispersed well in the nanocomposites, and the crystallization and mechanical properties of PLLA were enhanced clearly despite different preparation methods. ${ }^{17-19}$

However, there are not enough studies on biodegradable PPDO/POSS nanocomposites, and then PPDO/ome-POSS nanocomposites were prepared with various ome-POSS loading ranging from 1 to $10 \mathrm{wt} \%$ via the simple solution casting method in this work. The effect of ome-POSS on the crystallization, dynamic mechanical properties and morphology of PPDO were studied in detail. 


\section{Experiments}

\subsection{Materials and sample preparation}

PPDO was synthesized in our laboratory and the viscosity of PPDO was about $1.5 \mathrm{dl} \mathrm{g}^{-1}$ as determined by the intrinsic viscosity method. Ome-POSS was purchased from SigmaAldrich (Shanghai). For the fabrication of PPDO/ome-POSS nanocomposites, PPDO was mixed with 1, 5 and $10 \mathrm{wt} \%$ ome-POSS using the hexafluoroisopropanol as the mutual solvent. PPDO was dissolved in hexafluoroisopropanol and ome-POSS was separately dispersed in hexafluoroisopropanol. Then, the PPDO solution and ome-POSS solution were mixed together with the help of magnetic stirring and ultrasonic treatment for $1 \mathrm{~h}$. Finally, the solution was poured into a dish to evaporate the solvent at room temperature for $24 \mathrm{~h}$ and dried under vacuum at $40^{\circ} \mathrm{C}$ for $48 \mathrm{~h}$.

\subsection{Characterization}

2.2a Scanning electron microscopy (SEM): The morphology of the fracture surfaces of PPDO and its nanocomposites was observed by SEM (S-520, Hitachi, Japan). The samples were molded into film at $140^{\circ} \mathrm{C}$ and fractured in liquid nitrogen.

2.2b Differential scanning calorimetry (DSC): Thermal analysis was carried out with the DSC (Q20, TA-Instruments, USA). All the samples were heated to $140^{\circ} \mathrm{C}$ and held for $5 \mathrm{~min}$ to erase all the thermal history, and then cooled to $-40^{\circ} \mathrm{C}$ at a rate of $10^{\circ} \mathrm{C} \mathrm{min}^{-1}$. After that all the samples were heated to $140^{\circ} \mathrm{C}$ at a rate of $10^{\circ} \mathrm{C} \mathrm{min}^{-1}$. Isothermal crystallization was also performed with the DSC (Q20, TAInstruments, USA). The samples were heated to $140^{\circ} \mathrm{C}$ at $10^{\circ} \mathrm{C} \mathrm{min}^{-1}$ and held for $5 \mathrm{~min}$ to erase all the thermal history. Then they were cooled to the crystallization temperature $\left(55,65,70\right.$ and $\left.75^{\circ} \mathrm{C}\right)$ at $80^{\circ} \mathrm{C} \mathrm{min}^{-1}$ and held for certain time to get full crystallization.

2.2c X-ray diffraction $(X R D)$ : The XRD was performed with Philips X Pert Pro X-ray diffractometer. All the samples were pressed into films with a thickness of around $0.5 \mathrm{~mm}$ on a hot stage at $140^{\circ} \mathrm{C}$.

\section{2d Polarized optical microscopy (POM): A Changfang} polarizing optical microscope with a hot stage was used to study the crystallinity and spherulitic morphology of PPDO. The samples were heated to $140^{\circ} \mathrm{C}$ for $5 \mathrm{~min}$, and then cooled to $80^{\circ} \mathrm{C}$ to crystallize.

2.2e Dynamic mechanical analysis (DMA): The DMA was conducted on Q800 (TA-Instruments-Waters, USA) under tensile mode. The dimension of the specimen was $35 \mathrm{~mm} \times 5.0 \mathrm{~mm} \times 0.5 \mathrm{~mm}$ (length $\times$ width $\times$ thickness). The temperature range was from -60 to $80^{\circ} \mathrm{C}$ at heating rate of $3^{\circ} \mathrm{C} \mathrm{min}-1$ and at a frequency of $10 \mathrm{~Hz}$ under nitrogen atmosphere.

2.2f Self-nucleation experiments: The procedure followed was suggested by Fillon et $a l^{20}$ to study the selfnucleation behaviour of PPDO and PPDO/ome-POSS nanocomposite with the DSC (Q20, TA-Instruments, USA). The following consecutive steps were performed:

(a) the sample was first melted at $140^{\circ} \mathrm{C}$ for $5 \mathrm{~min}$, to erase all previous thermal history of PPDO;

(b) the sample was then cooled to $50^{\circ} \mathrm{C}$ at $10^{\circ} \mathrm{C} \mathrm{min}^{-1}$ with the aim of creating a standard thermal history;

(c) the sample was again heated at the same rate up to a temperature denoted $T_{\mathrm{s}}$ or self-nucleation temperature;

(d) the sample was held at $T_{\mathrm{s}}$ for $5 \mathrm{~min}$;

(e) a cooling scan of the sample was recorded from $T_{\mathrm{s}}$ down to $0^{\circ} \mathrm{C}$; and

(f) finally, a heating scan of the sample from $0^{\circ} \mathrm{C}$ up to $140^{\circ} \mathrm{C}$ was recorded to register the melting behaviour.

\section{Results and discussion}

\subsection{Dispersion of ome-POSS on the PPDO matrix}

It is well known that the dispersion of POSS in the polymer matrix may influence the physical properties of biodegradable polymer, and then the dispersion of ome-POSS in the PPDO matrix was studied by the SEM. Figure 1 shows the SEM images of fracture surface of PPDO and its nanocomposites. Several white particles with the size of about 100$400 \mathrm{~nm}$, corresponding to the aggregation of ome-POSS, are randomly dispersed in the PPDO matrix suggesting that omePOSS is well dispersed in the PPDO matrix. The aggregation of ome-POSS is not serious even with loading of $10 \mathrm{wt} \%$ ome-POSS with the dimensions of about 100-400 $\mathrm{nm}$. It could be noted that the dispersion of ome-POSS is better in PPDO via the solution casting method than PP and $\mathrm{PE}$, which were prepared via melt compounding. ${ }^{15,16}$
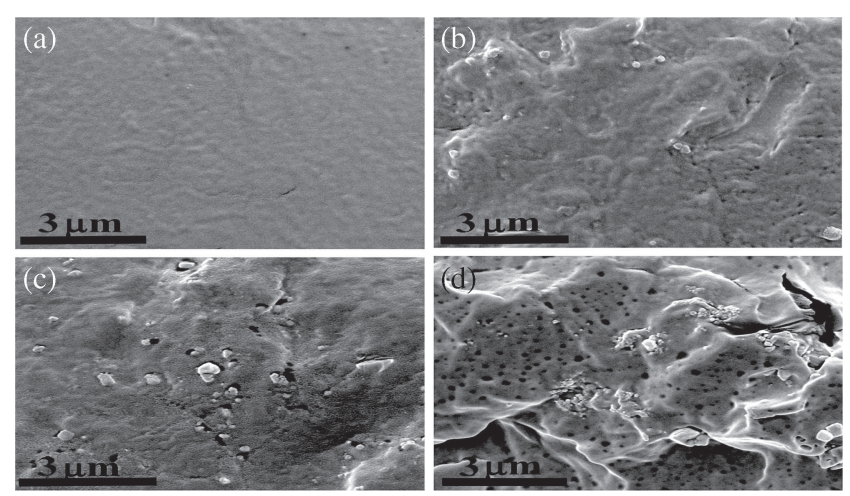

Figure 1. SEM images showing an overall morphology of fracture surface of (a) PPDO, (b) ome-POSS $1 \mathrm{wt} \%$, (c) ome-POSS 5 $\mathrm{wt} \%$ and (d) ome-POSS $10 \mathrm{wt} \%$. 
However, there contains evidence of phase-separated omePOSS domains (figure 1c and d), indicating that there is low compatibility between ome-POSS and the PPDO matrix. Such low-compatibility is a result of the strong interaction between the methyl group of ome-POSS and the PPDO matrix. In brief, the solution casting method is expected to provide a better dispersion of ome-POSS in the matrix which may be due to the lower viscosity surrounding with the help of solvent than the melt compounding technique.

\subsection{Thermal behaviour of PPDO/ome-POSS blend}

It is essential to understand the thermal behaviour of the nanocomposites, and then the DSC was used to analyse the thermal behaviour of PPDO/ome-POSS nanocomposites. Figures 2 and 3 show the cooling and heating scans at $10^{\circ} \mathrm{C} \mathrm{min}^{-1}$ for PPDO and PPDO/ome-POSS blends after keeping them at $140^{\circ} \mathrm{C}$ for $5 \mathrm{~min}$ to erase their thermal history. All of the relevant data from figures 2 and 3 are listed in tables 1 and 2 .

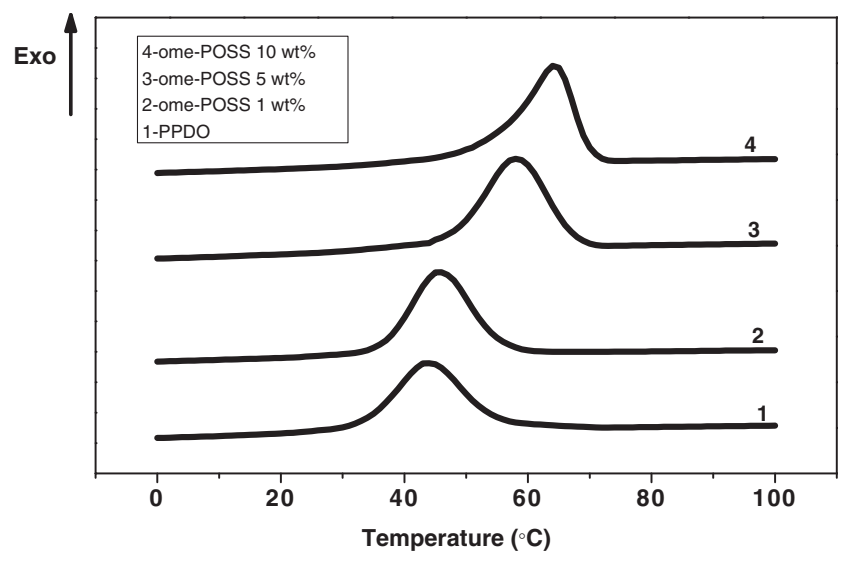

Figure 2. DSC cooling scans for neat and filled PPDO after erasing thermal history at $140^{\circ} \mathrm{C}$ for $5 \mathrm{~min}$, cooling rate: $10^{\circ} \mathrm{C} \mathrm{min}{ }^{-1}$.

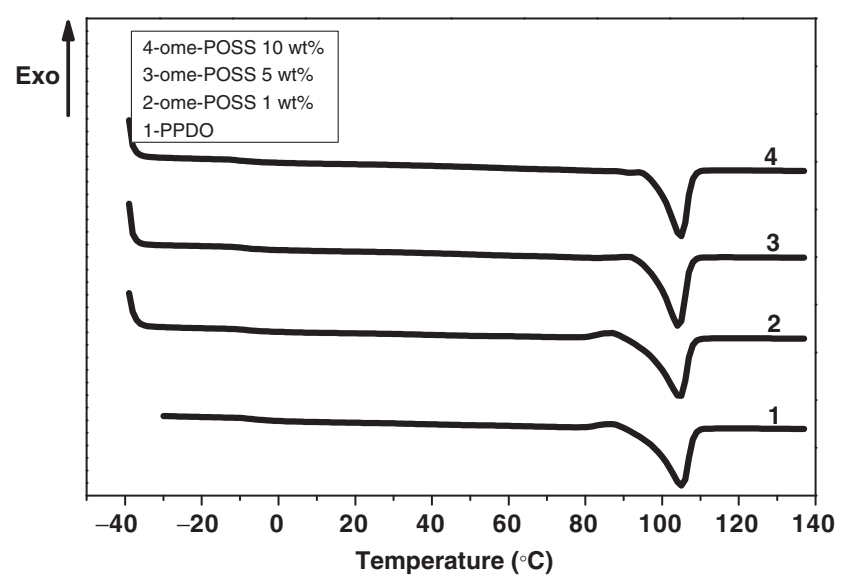

Figure 3. DSC subsequent heating scans after the cooling runs in figure 1 for neat and filled PPDO, heating rates: $10^{\circ} \mathrm{C} \mathrm{min}^{-1}$.
The cooling scans of neat PPDO show a crystallization exothermal peak around $43^{\circ} \mathrm{C}$ (table 1 ). It shows a clearly nucleating affect to PPDO when 1 wt\% ome-POSS was added to PPDO, which cause several effects, including a substantial increase of the onset and peak crystallization temperatures (table 1). It is reported that the nucleation density is related to the dynamic peak crystallization temperature during cooling from melt $\left(T_{\text {peak }}\right)$, and it is also demonstrated that as the peak temperature $\left(T_{\text {peak }}\right)$ increases the number of nuclei per unit volume also increases. The $T_{\text {oneset }}$ and $T_{\text {peak }}$ were increased with the increase in ome-POSS loading, indicating that the nucleating effect was enhanced with the increase in ome-POSS loading. The largest variation on crystallization temperatures occurred at the ome-POSS content of $10 \%$. It shows that ome-POSS can act as a high-performance nuclear agent for PPDO. In order to estimate the nucleating efficiency of ome-POSS, the efficiency scale proposed by Fillon was employed. ${ }^{8}$ The efficiency scale is used as a reference not for the neat polymer but for the polymer that was self-nucleated to saturate.

The nucleating efficiency put forward by Fillion can be defined as follows:

$$
\mathrm{NE}=\left(T_{\mathrm{cNA}}-T_{\mathrm{c}}\right) /\left(T_{\mathrm{cmax}}-T_{\mathrm{c}}\right),
$$

where $T_{\mathrm{cNA}}$ is the peak crystallization temperature of the polymer with the nucleating agent to be evaluated, $T_{\mathrm{c}}$ the peak crystallization temperature of the neat polymer, and $T_{\text {cmax }}$ the optimum self-nucleating temperature of the neat polymer. NE is expressed as a percentage where 0 represents no nucleating action and $100 \%$ the ideal nucleating action.

By calculation, the NE value of $1 \mathrm{wt} \%$ ome-POSS was only $4.75 \%\left(T_{\mathrm{cmax}}\right.$ of PPDO is $\left.90.8^{\circ} \mathrm{C}\right),{ }^{5}$ meanwhile the NE values of 30.75 and $44.29 \%$ were achieved for blends with 5 and $10 \mathrm{wt} \%$ ome-POSS, respectively. Compared

Table 1. DSC data of PPDO/ome-POSS blends from cooling scans.

\begin{tabular}{lccccc}
\hline Blend & $\begin{array}{c}\text { Blend } \\
\text { ratio }\end{array}$ & $\begin{array}{c}T_{\text {onset }} \\
\left({ }^{\circ} \mathrm{C}\right)\end{array}$ & $\begin{array}{c}T_{\text {peak }} \\
\left({ }^{\circ} \mathrm{C}\right)\end{array}$ & $\begin{array}{c}H_{\mathrm{c}} \\
\left(\mathrm{J} \mathrm{g}^{-1}\right)\end{array}$ & $\begin{array}{c}\mathrm{NE} \\
(\%)\end{array}$ \\
\hline PPDO & $100 / 0$ & 54.35 & 43.25 & 42.43 & - \\
PPDO/ome-POSS & $99 / 01$ & 55.30 & 45.51 & 45.44 & 4.75 \\
& $95 / 05$ & 67.48 & 57.87 & 51.32 & 30.75 \\
& $90 / 10$ & 70.04 & 64.31 & 49.20 & 44.29 \\
\hline
\end{tabular}

Table 2. DSC data of PPDO/ome-POSS blends from heating scans.

\begin{tabular}{lcccccc}
\hline & $\begin{array}{c}T_{\mathrm{g}} \\
\left({ }^{\circ} \mathrm{C}\right)\end{array}$ & $\begin{array}{c}T_{\mathrm{cc}} \\
\left({ }^{\circ} \mathrm{C}\right)\end{array}$ & $\begin{array}{c}\Delta H_{\mathrm{cc}} \\
\left(\mathrm{J} \mathrm{g} \mathrm{g}^{-1}\right)\end{array}$ & $\begin{array}{c}T_{\mathrm{m}} \\
\left({ }^{\circ} \mathrm{C}\right)\end{array}$ & $\begin{array}{c}\Delta H_{\mathrm{m}} \\
\left(\mathrm{J} \mathrm{g}^{-1}\right)\end{array}$ & $\begin{array}{c}D_{\mathrm{t}} \\
(\%)\end{array}$ \\
\hline PPDO & -10.18 & 85.68 & 3.04 & 104.99 & 52.92 & 35.33 \\
$1 \%$ ome-POSS & -8.73 & - & - & 104.54 & 56.21 & 40.22 \\
$5 \%$ ome-POSS & -9.66 & - & - & 104.28 & 54.65 & 40.76 \\
$10 \%$ ome-POSS & -9.69 & - & - & 104.85 & 49.50 & 38.97 \\
\hline
\end{tabular}


to $\mathrm{BN}$, talc and HA nanocomposites, ${ }^{5}$ the nucleating efficiency of PPDO/ome-POSS is large. The NE value increases with the increase in ome-POSS, indicating the high performance of ome-POSS on the nanocomposites. It shows a clear nucleating effect of ome-POSS

$$
D_{\mathrm{t}}=H_{\mathrm{m}} /\left(W \Delta H_{\mathrm{m}}^{\mathrm{o}}\right) \text {. }
$$

$D_{\mathrm{t}}$ is the degree of crystallinity of the blends, $\Delta H_{\mathrm{m}}$ the enthalpy of melting per gram of blend, and $W$ the mass fraction of PPDO in the blend. The enthalpy of melting $\Delta H_{\mathrm{m}}^{\circ}$ for $100 \%$ crystalline PPDO was determined to be $141.18 \mathrm{~J} \mathrm{~g}^{-1}$.

Although the ether bond confers high flexibility to PPDO, it does not crystallize completely during cooling from the melt and shows a cold crystallization exothermal peak $\left(T_{\mathrm{cc}}\right)$ during subsequent heating in the DSC. The cold crystallization exothermal peak of neat PPDO was observed at $86^{\circ} \mathrm{C}$, indicating that the material did not completely crystallize during the previous cooling at $10^{\circ} \mathrm{C} \mathrm{min}^{-1}$. It is a process of recrystallization during the heating process when the temperature is above the $T_{\mathrm{g}}$. In the present study, it is observed that because the PPDO/ome-POSS blend crystallized quite perfectly representing the higher values of crystallinity (table 2), they did not show cold crystallization exothermal peak. The degree of crystallinity of PPDO increases when ome-POSS was added to the PPDO matrix.

\subsection{Effect of ome-POSS on the isothermal melt crystallization of $P P D O$}

It is interesting to study the effect of ome-POSS on the crystal structure of PPDO in the PPDO/ome-POSS nanocomposites. XRD experiments were performed to study the crystal structure of neat PPDO and its nanocomposites at different ome-POSS contents. In figure $4 \mathrm{a}$, the presence of a number of strong diffraction peaks shows that ome-POSS are highly crystalline. ${ }^{18}$ The crystalline of ome-POSS also appears in the PPDO/ome-POSS nanocomposites, suggesting ome-POSS may exist as separate crystal or particles are able to crystallize when they are dispersed in the PPDO matrix. Neat PPDO shows two main diffraction peaks at around $21.85^{\circ}$ and $23.45^{\circ},{ }^{21}$ moreover, the two strong sharp diffraction peaks also appear in the nanocomposites indicating that the crystal structure of PPDO remains unchanged with ome-POSS. However, the diffraction peak's intensity of PPDO in the nanocomposites is stronger than neat PPDO, indicating that the presence of ome-POSS may enhance the crystallinity of PPDO. In brief, the crystal structure of PPDO keeps unchanged despite the addition of ome-POSS in the PPDO matrix.

Spherulitic morphology of neat PPDO and its nanocomposite was studied with POM. Figure 5 is the spherulitic morphology of neat PPDO and $10 \mathrm{wt} \%$ ome-POSS nanocomposite crystallized at $80^{\circ} \mathrm{C}$. As shown from the figure, neat PPDO and $10 \mathrm{wt} \%$ ome-POSS nanocomposite form banded spherulites that can exhibit a well-defined Maltese cross. It is obvious that the number of PPDO spherulites is larger in the PPDO/ome-POSS nanocomposite than in neat PPDO, moreover, the size of PPDO spherulites is smaller in the PPDO/ome-POSS nanocomposite than in neat PPDO. Such observations show that the nucleation density of PPDO spherulites increases dramatically in the PPDO/ome-POSS nanocomposite because of the nucleating effect of omePOSS. In all, the loadings of ome-POSS in the PPDO matrix are the main factors which influence the spherulite morphology, and ome-POSS can act as nucleating agent for PPDO.

The ome-POSS can act as nuclear agent for PPDO, which is estimated to improve the crystallization rate of PPDO. The isothermal crystallization behaviour of neat PPDO and its

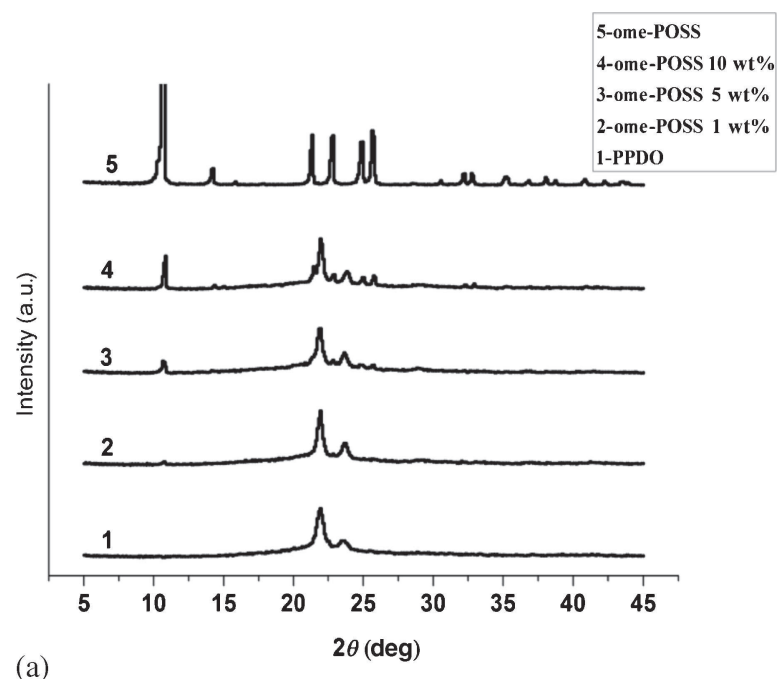

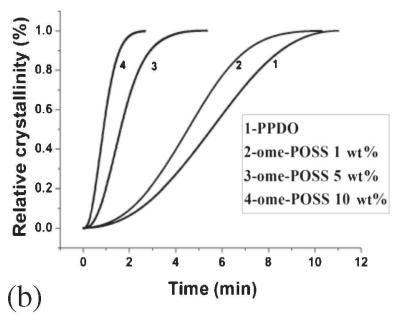

(b)

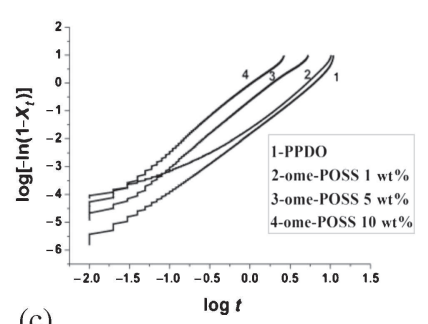

(c)

Figure 4. (a) XRD patterns of neat PPDO and its nanocomposites, (b) variation in relative crystallinity with crystallization time for neat PPDO and its nanocomposites at $70^{\circ} \mathrm{C}$ and (c) the related Avrami plots. 

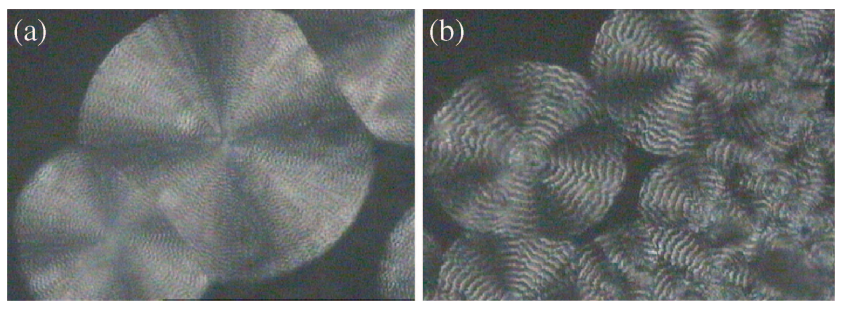

Figure 5. POM images of neat PPDO and its nanocomposite crystallized at $80^{\circ} \mathrm{C}$ : (a) neat PPDO and (b) $10 \mathrm{wt} \%$ ome-POSS loading nanocomposite.

nanocomposites were studied with DSC at different crystallization times from 55 to $75^{\circ} \mathrm{C}$. Figure $4 \mathrm{~b}$ shows the plots of relative crystallinity against crystallization time at $70^{\circ} \mathrm{C}$. From figure $4 \mathrm{~b}$ it is clear that all these curves have the same sigmoid shape and the corresponding crystallization time for the nanocomposites decrease with the increase in ome-POSS contents. It is apparent that the incorporation of ome-POSS can enhance the isothermal melt crystallization of PPDO when compared to neat PPDO. The isothermal crystallization of PPDO is faster and increases with the increase in ome-POSS content.

Figure $4 \mathrm{c}$ shows the Avrami plots of neat PPDO and its nanocomposites. The Avrami equation can be used to analyse the isothermal crystallization of neat PPDO and its nanocomposites $^{5-7}$

$$
1-X_{t}=\exp \left(-k t^{n}\right)
$$

where $X_{t}$ is the relative degree of crystallinity at crystallization time $(t), n$ the Avrami exponent depending on the nature of nucleation and growth geometry of the crystals, and $k$ the crystallization rate

$$
t_{1 / 2}=(\ln 2 / k)^{1 / n} .
$$

All the relevant data are listed in table 3. It can be seen that the Avrami exponents of neat PPDO and its nanocomposites are between 2 and 3 despite the addition of ome-POSS. Since $n \leq 3$ was found at such low temperature, the results may indicate the formation of axialites or other type of twodimensional lamellar aggregates, although it was unable to observe them. However, there are many previous studies in the literature that have reported the formation of spherulites in the case of Avrami indexes of lower than 3. ${ }^{6,7,9}$ The $k$ data are listed in table 1; however, it is difficult to analyse the overall crystallization rate directly from $k$ values. Thus, the crystallization half-time $\left(t_{1 / 2}\right)$, the time required to achieve $50 \%$ of the final crystallinity of the samples, is introduced to analyse the crystallization kinetics. The $t_{1 / 2}$ is increasing with the increase in $T_{\mathrm{c}}$, indicating the crystallization rate of neat PPDO and its nanocomposites decreases with the increase in $T_{\mathrm{c}}$. Such results are reasonable since it is difficult for the samples to nucleate at high $T_{\mathrm{c}}$, thereby resulting in the decreasing crystallization rate. The $t_{1 / 2}$ is smaller in the nanocomposites than neat
Table 3. Isothermal crystallization kinetics parameters of neat PPDO and its nanocomposites at different $T_{\mathrm{c}} \mathrm{s}$ based on the Avrami equation.

\begin{tabular}{lllc}
\hline Temperature $\left({ }^{\circ} \mathrm{C}\right)$ & \multicolumn{1}{c}{$n$} & \multicolumn{1}{c}{$k$} & $t_{1 / 2}$ \\
\hline PPDO & & & \\
55 & 2.377 & 0.869621 & 0.908998 \\
65 & 2.70664 & 0.040041 & 2.867569 \\
70 & 2.17518 & 0.016672 & 5.244473 \\
75 & 2.5926 & 0.002688 & 9.058949 \\
& & & \\
$1 \%$ ome-POSS & & & \\
55 & 2.67825 & 0.947545 & 0.889825 \\
65 & 2.45583 & 0.088861 & 2.308151 \\
70 & 2.17002 & 0.030499 & 4.218186 \\
75 & 2.01 & 0.015849 & 6.551353 \\
& & & \\
$5 \%$ ome-POSS & & & 0.607175 \\
55 & 2.5635 & 2.34053 & 1.037158 \\
65 & 2.33864 & 0.636458 & 1.706961 \\
70 & 2.22878 & 0.210499 & \\
75 & 2.0036 & 0.0889 & 2.787157 \\
$10 \%$ ome-POSS & & & \\
55 & & & 0.905326 \\
65 & 1.867 & 0.834584 & 0.484048 \\
70 & 2.58002 & 4.506299 & 0.927557 \\
75 & 2.2837 & 0.823019 & \\
\hline & 2.545 & 0.119674 & 0.99501 \\
\hline
\end{tabular}

PPDO at given $T_{\mathrm{c}}$ indicating that the isothermal melt crystallization of PPDO is accelerated with the help of ome-POSS. Such results show ome-POSS can act as a nucleating agent during the isothermal crystallization of PPDO in the PPDO/ome-POSS nanocomposites. In table 3, it is also shown that the $t_{1 / 2}$ decreases with the increase in ome-POSS loading in the PPDO/ome-POSS nanocomposites at given $T_{\mathrm{c}}$, suggesting that the ome-POSS has a significant effect on the crystallization of PPDO. In all, the overall crystallization rate is accelerated with the addition of ome-POSS to the PPDO matrix, and the enhancement of crystallization rate of PPDO is affected by the content of ome-POSS.

\subsection{Self-nucleation of PPDO and PPDO/ome-POSS nanocomposite}

Figure 6 shows DSC scans corresponding to dynamic cooling at $10^{\circ} \mathrm{C} \mathrm{min}-1$ of PPDO after self-nucleation at the indicated $T_{\mathrm{s}}$ temperature. Subsequent heating scans after cooling from $T_{\mathrm{S}}$ are shown in figure 7 . To analyse this data, the relevant data as a function of self-nucleation temperature $T_{\mathrm{s}}$ in figures 8 and 9 have been plotted.

Figure 6 shows three domains clearly. In view of figure 6 , the cooling runs for $T_{\mathrm{s}} \geq 116^{\circ} \mathrm{C}$ are identical to that of $T_{\mathrm{s}}=140^{\circ} \mathrm{C}$. The peak crystallization and enthalpy do not change with temperature when $T_{\mathrm{s}}$ is greater than $116^{\circ} \mathrm{C}\left(T_{\mathrm{s}} \geq\right.$ $116^{\circ} \mathrm{C}$ ) (figure 8). A similar situation is found with the heating scans, the heating runs are identical to that of $T_{\mathrm{s}}=140^{\circ} \mathrm{C}$ 


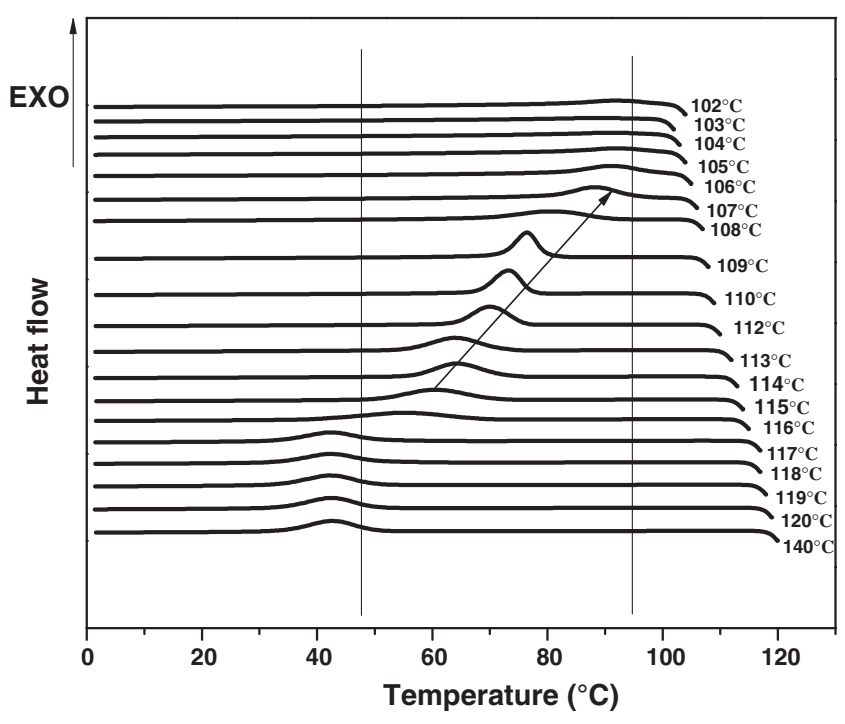

Figure 6. DSC cooling scans at $10^{\circ} \mathrm{C} \mathrm{min}^{-1}$ of PPDO after $5 \mathrm{~min}$ at the indicated $T_{\mathrm{S}}$ temperature.

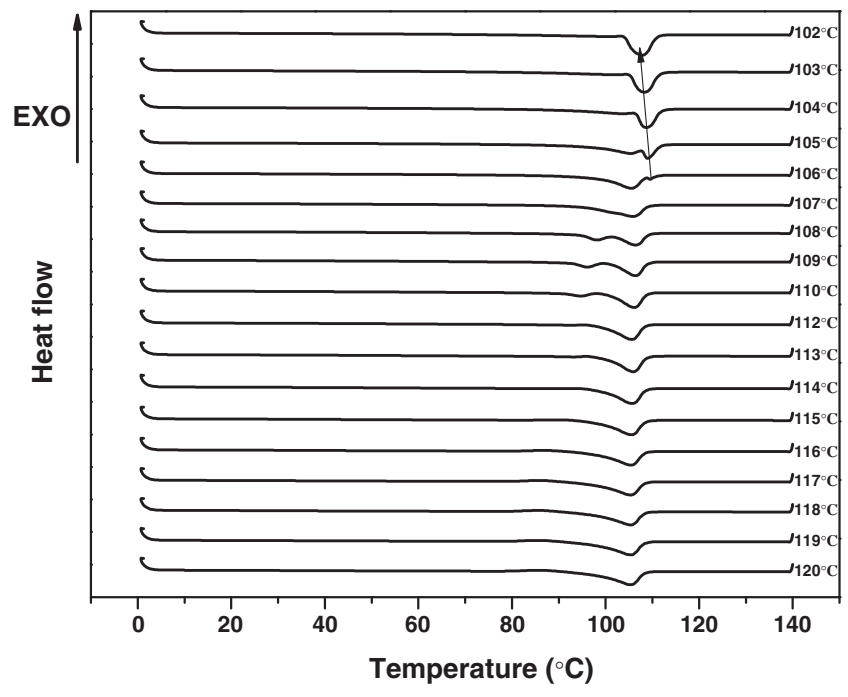

Figure 7. Subsequent DSC heating scans at $10^{\circ} \mathrm{C} \mathrm{min}^{-1}$ of PPDO after cooling as shown in figure 1.

when $T_{\mathrm{S}}$ is greater than $116^{\circ} \mathrm{C}$. The melting point is invariant with $T_{\mathrm{s}}$, as shown in figure 9 , when $T_{\mathrm{s}}$ is greater than $116^{\circ} \mathrm{C}$. This shows when $T_{\mathrm{s}}$ is greater than $116^{\circ} \mathrm{C}$, the time spent at $T_{\mathrm{s}}$ is enough to erase the crystalline memory of the material, then the PPDO needed the maximum undercooling in order to crystallize when cooled from $T_{\mathrm{S}}$ and the crystallization temperature does not change with $T_{\mathrm{s}}$ in that domain. The behaviour of Domain I is that of PPDO at $T_{\mathrm{s}} \geq 116^{\circ} \mathrm{C}$. In that domain only heat-resistant heterogeneous nuclei can survive and are able to nucleate the polymer once it is cooled to the appropriate temperature.

An obvious change in the nucleation behaviour of PPDO is found when $T_{\mathrm{s}}$ lower than $116^{\circ} \mathrm{C}$ are used as indicated in figure 8. This region is self-nucleation domain (Domain II). In this $T_{\mathrm{s}}$ region, PPDO is partial melting and leaving

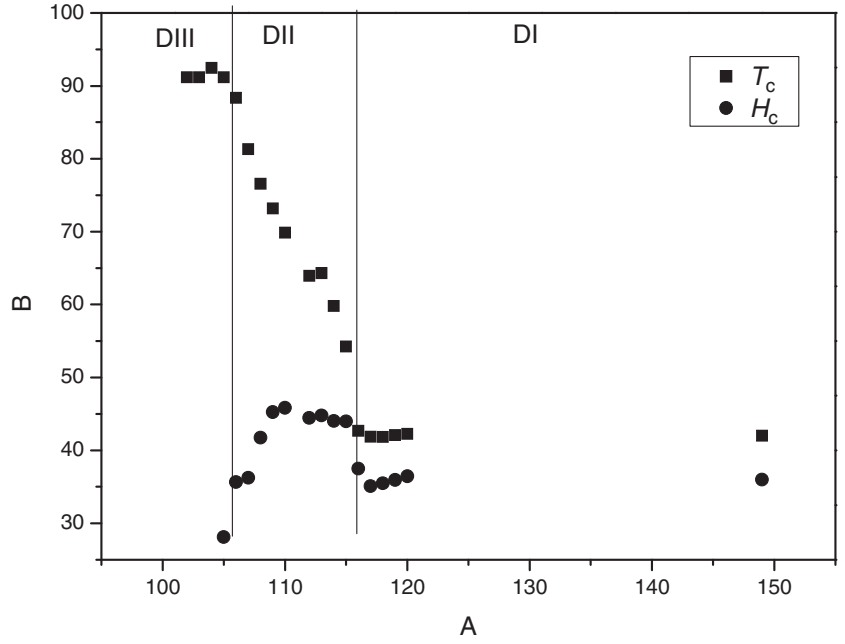

Figure 8. Peak crystallization temperature and crystallization enthalpy as a function of self-nucleation temperature for PPDO.

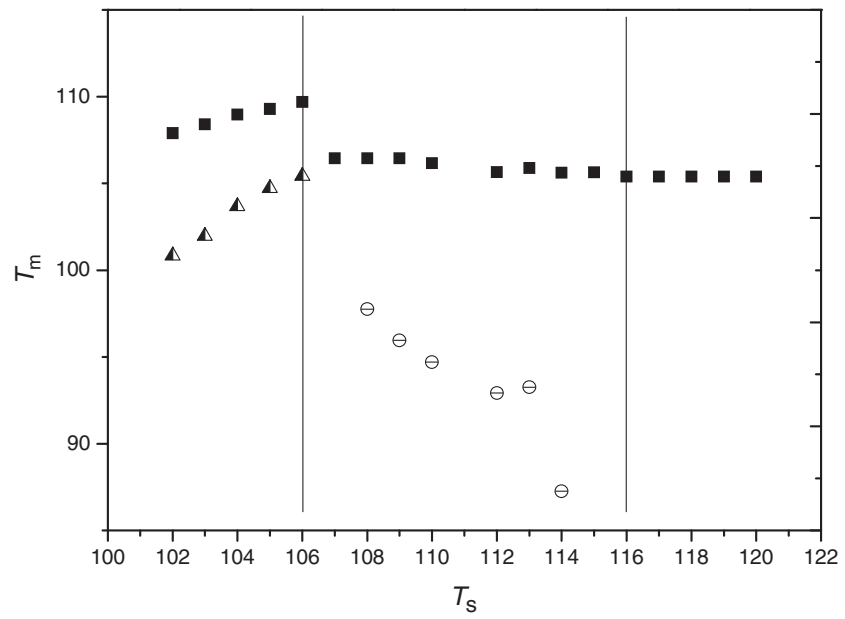

Figure 9. Melting temperature of self-nucleation temperature for PPDO.

small crystal fragments which is too small to anneal, but big enough to act as self-nuclei. The practical consequence is an enormous increase in nucleation density as $T_{\mathrm{S}}$ is lower within the limits of Domain II. Self-nucleation causes two major effects in the cooling scans of PPDO from melt. The first effect is the decrease in the undercooling needed for crystallization as $T_{\mathrm{s}}$ is decreased (the peak crystallization decrease with the increase in $T_{\mathrm{s}}$ ), which is shown in figures 6 and 8 . The second effect is the enthalpy of crystallization in that domain is bigger than others as shown in figure 8. A similar behaviour has been seen in PET within Domain II, ${ }^{22}$ and this effect can negligible since the crystallization rate is extremely fast for iPP. Figure 9 shows the heating scans after the crystallization from $T_{\mathrm{s}}$. For those temperature located within Domain II $\left(116-106^{\circ} \mathrm{C}\right)$ or the self-nucleation domain, the lack of annealing of unmelted crystals can be seen in the heating scans of figure 7 , then there are no higher melting 
endotherms that exhibited by the material in Domain I. However, there are two melting endotherms for all the samples in Domain II (figures 7 and 9). The first melting temperature is highly dependent on the $T_{\mathrm{s}}$ value and occurs at lower temperature which decrease with the increase in $T_{\mathrm{s}}$, while the second one is independent of $T_{\mathrm{s}}$ and occurs at the same temperature as shown in figure 9. The lower temperature melting points is the fusion of the crystals formed during cooling from $T_{\mathrm{s}}$, while the higher temperature one is due to the fusion of crystals that were partially melted and recrystallized during scans which is why its $T_{\mathrm{m}}$ values are independent of $T_{\mathrm{s}}$.

Finally, if the $T_{\mathrm{s}}$ decrease below $106^{\circ} \mathrm{C}$, Domain III is reached. In this region, partial melting takes place but the amount of unmelted material is large enough to experience annealing during the holding time at $T_{\mathrm{s}}$, therefore, the transition to Domain III can be detected in the subsequent heating scans after cooling from the $T_{\mathrm{s}}$. As a result of annealing and self-nucleation, there are two endotherm peaks in the heating scans (figure 7). Appearance of high temperature (the arrow) and a small peak are the hallmark of Domain III.

The heating runs of figure 7 in Domain III show complex endotherms that are composed of two signals: the one endotherm that corresponds to the fusion of the crystals that were annealed at $T_{\mathrm{s}}$, and the other corresponds to the fusion of the crystals formed during cooling. Figure 9 shows how these two $T_{\mathrm{m}}$ values depend on $T_{\mathrm{s}}$ within Domain III. The high melting temperature is independent of $T_{\mathrm{s}}$ (figure 9), which corresponds to the fusion the crystals formed during cooling and altered during the heating scan by partial melting and recrystallization process. The melting point which is a linear function of $T_{\mathrm{s}}$ is clearly the one corresponding to the fusion of annealed crystals. ${ }^{5,20}$

The self-nucleation experimental protocol was applied to PPDO with 5\% ome-POSS under study. The results are plotted in figure 10 . When $5 \%$ ome-POSS was added to PPDO, there also appear three domains. For ome-POSS

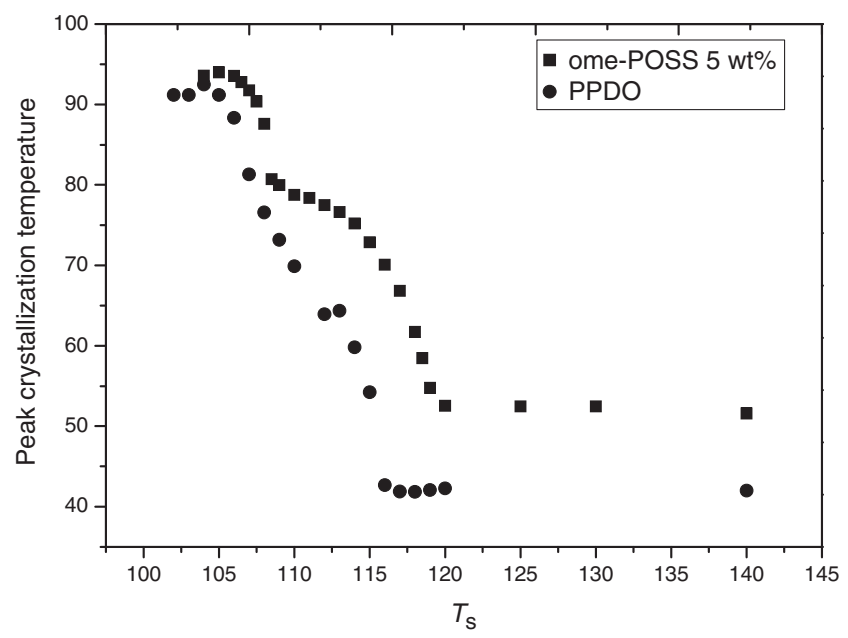

Figure 10. Peak crystallization temperatures as a function of self-nucleation temperature for neat PPDO and 5\% ome-POSS nanocomposite. nanocomposite, Domain I is $T_{\mathrm{s}} \geq 120^{\circ} \mathrm{C}$ and Domain II is between 106 and $120^{\circ} \mathrm{C}$, meanwhile the Domain III is $T_{\mathrm{s}} \leq 106^{\circ} \mathrm{C}$. From XRD, it is shown that ome-POSS may exist as separate crystal or ome-POSS is able to crystallize when it is dispersed in the PPDO matrix. In view of PPDOs self-nucleation, the unmelted PPDO crystals can act as nuclei, meanwhile the crystallized nanoparticles (omePOSS) can also act as nuclei, and then there appear several effects. In Domain I, only temperature-resistant nuclei can survive, and then the temperature of Domain $\mathrm{I}$ is $T_{\mathrm{s}} \geq 116^{\circ} \mathrm{C}$ for neat PPDO. However, temperature-resistant nuclei and ome-POSS nuclei coexist in PPDO/ome-POSS nanocomposite, then the temperature of Domain $\mathrm{I}$ is larger than $120^{\circ} \mathrm{C}$ and the peak crystallization temperature is bigger than neat PPDOs. As a result of lack of unmelted PPDO crystals, the neat PPDO can melt completely when the temperature is above $116^{\circ} \mathrm{C}$. However, the crystallized ome-POSS can act as nuclei, and then the PPDO in nanocomposite can show self-nucleation behaviour when the temperature is between 116 and $120^{\circ} \mathrm{C}$. In Domain II, as a result of PPDO crystals and crystallized ome-POSS nuclei, the peak crystallization temperature of ome-POSS nanocomposite is larger than neat PPDOs. Therefore, the crystallized nanoparticle can act as nuclei to improve the crystallization rate of PPDO.

\subsection{Effect of ome-POSS on the dynamic mechanical properties}

Figure 11 shows the dynamic mechanical properties of neat PPDO and its nanocomposites. Figure 11 shows the temperature dependence of storage modulus $\left(E^{\prime}\right)$ and loss modulus $\left(E^{\prime \prime}\right)$ of neat PPDO and its nanocomposites. From figure $11 \mathrm{a}$, it is obvious that the $E^{\prime}$ increase after the addition of ome-POSS at low temperature in the glass state $\left(-60\right.$ to $\left.-10^{\circ} \mathrm{C}\right)$, suggesting the improved reinforcement effect with the ome-POSS particles. The storage modulus of neat PPDO is around $6000 \mathrm{MPa}$ at $-60^{\circ} \mathrm{C}$, while the $E^{\prime}$ increases to around $6800,6200,7000 \mathrm{MPa}$, respectively, with the increase in the ome-POSS loading from 1,5 and $10 \%$ in the PPDO matrix. The $E^{\prime}$ increases apparently with only $1 \%$ ome-POSS, and the $E^{\prime}$ is significantly improved to $7000 \mathrm{MPa}$ when $10 \%$ ome-POSS is added to PPDO. The obvious improvement in the $E^{\prime}$ could be attributed to the combined effect of high performance and well dispersion of ome-POSS in the PPDO matrix. Similar improvement was also found in other biomaterials. ${ }^{17-19}$ Moreover, both neat PPDO and its nanocomposites exhibit a sharp reduction of elastic modulus around $20^{\circ} \mathrm{C}$, corresponding to the glass transition of PPDO. In addition, the $E^{\prime}$ of neat PPDO and its nanocomposites are small and tend to zero eventually from $80^{\circ} \mathrm{C}$ (figure $11 \mathrm{a}$ ), indicating that the nanocomposites stiffness become matrix dependent at the rubbery state. The glass transition of neat PPDO and nanocomposites is similar estimating at $20^{\circ} \mathrm{C}$, indicating that the existence of ome-POSS does not show pronounced affect of the segment motion of PPDO in the nanocomposites. The ome-POSS can enhance 


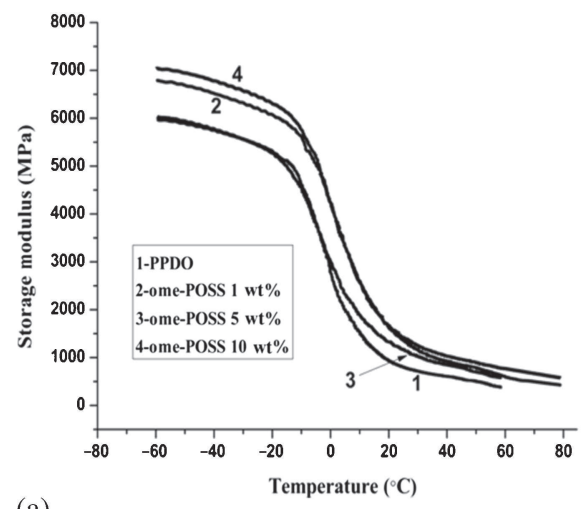

(a)

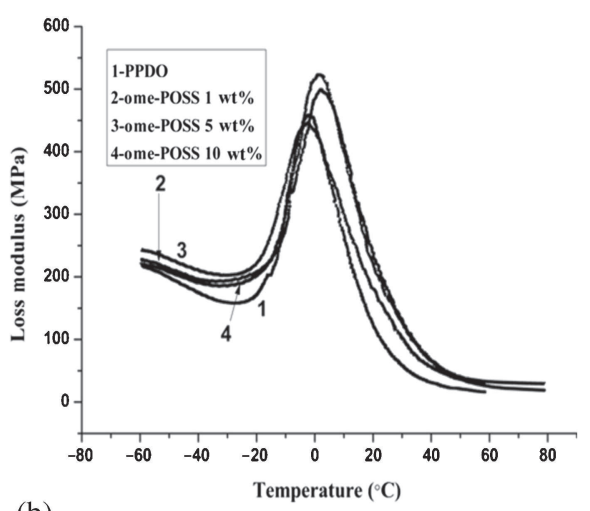

(b)

Figure 11. Temperature dependence of (a) storage modulus and (b) loss modulus for neat PPDO and its nanocomposites.

the crystallization rate and mechanical properties of PPDO, and hence it is hoped that it can be widely used as other medical devices.

\section{Conclusions}

Novel biodegradable PPDO/ome-POSS nanocomposites were prepared successfully via the simple solution casting method with various ome-POSS contents. Isothermal melt crystallization studies show that the overall crystallization rates are faster in nanocomposites than in neat PPDO and increase with the increase in the ome-POSS content, meanwhile the crystallization mechanism and crystal structure unchanged in the nanocomposites. SEM shows that omePOSS is well dispersed in the PPDO matrix, indicating that the solution casting method is an efficient way to prepare nanocomposites. It also enhances the dynamic mechanical property of PPDO in the nanocomposites with respect to neat PPDO, which may be of great interest for the practical application of PPDO.

\section{References}

1. Ke-Ke Y and Yu-Zhong W 2002 J. Macromol. Sci._Polym. Rev. C42 373

2. Yin-Qiao Z, Song-Song D, Yuan Y and Yu-Zhong W $2014 \mathrm{~J}$. Appl. Polym. Sci. 101

3. Pezzin A P T, van Ekenstein G O R and Duek E A R 2001 Polymer 428303

4. Zeng J-B, Srinivansan M, Narayan R and Wang Y-Z 2011 Ind. Eng. Chem. Res. 504471
5. Sabino M A, Ronca G and Muller A J 2000 J. Mater. Sci. 35 5071

6. Wang X L, Wang Y Z, Wang D Y and Yang Z 2004 Acta Mater. 524899

7. Zhang L L, Goh S H and Lee S Y 1998 Polymer 394841

8. Zhi-Xuan Z, Yu-Zhong W, Ke-Ke Y, Si-Chong C, Gang W et al 2006 Polym. Int. 55383

9. Ling-Xi Z, Ming-Jing L, Jian-Bing Z and Yu-Zhong W 2012 Polym. Chem.-UK 32537

10. Ke-Ke Y, Yan Z, Fang L, Fu-Yun H, Zhi-Cheng Q and YuZhong W 2009 J. Macromol. Sci. B 481031

11. Zhi-Cheng Q, Jing-Jing Z, Ying N, Cai-Li H, Ke-Ke Y and Yu-Zhong W 2013 Soft Mater. 1198

12. Baney R H, Itoh M, Sakakibara A and Suzuki T 1995 Chem. Rev. 951409

13. Kuo S-W and Chang F-C 2011 Prog. Polym. Sci. 361649

14. Li G Z, Wang L C and Pittman C U 2001 J. Inorg. Organomet. P. 11123

15. Fina A, Tabuani D, Frache A and Camino G 2005 Polymer 46 7855

16. Hato M J, Ray S S and Luyt A S 2008 Macromol. Mater. Eng. 293752

17. Pan H and Qiu Z 2010 Macromolecules 431499

18. Yu J and Qiu Z 2011 ACS Appl. Mater. Int. 3890

19. Zhaobin Q and Hong P 2010 Compos. Sci. Technol. 70 1089

20. Fillon B, Wittman J C, Lotz B and Thierry A 1993 J. Polym. Sci.-Polym. Phys. 311383

21. Pezzin A P T, van Ekenstein G, Zavaglia C A C, Ten Brinke G and Deuk E A R 2003 J. Appl. Polym. Sci. 882744

22. Molin C H, Mendez G A and Muller A J 1998 J. Appl. Polym. Sci. 701725 